\title{
4 Ethische Herausforderungen in der präklinischen Notfallmedizin
}

\author{
Heiner Krieter
}

\subsection{Einleitung}

Die Notfallmedizin in Deutschland hat ein im Vergleich zu anderen Ländern extrem hohes Niveau erreicht. Nirgends sonst auf der Welt findet sich ein rund um die Uhr flächendeckendes und arztbesetztes Notfallsystem. Neben den erweiterten therapeutischen Maßnahmen eröffneten sich in den vergangenen Jahren zahlreiche diagnostische Optionen, die noch am Einsatzort zuverlässige Entscheidungshilfen geben. Schließlich trägt die Weiterentwicklung des Berufsbilds vom Rettungsassistenten zum Notfallsanitäter dazu bei, dass Notfallpatienten bereits präklinisch von einem hoch qualifizierten Rettungsteam fachlich kompetent betreut werden können. Während die technischen, medizinischen und personellen Standards wohldefiniert sind, fehlen derzeit noch weitgehend vergleichbare Standards und Handlungsempfehlungen für ethische Fragen. Dies verwundert umso mehr, als gerade in der Notfallmedizin kritische Entscheidungen bei lebensbedrohlichen Erkrankungen eher die Regel als die Ausnahme sind. Diese Entscheidungen werden zudem stets unter hohem Zeitdruck getroffen, sodass es wichtig und hilfreich wäre, neben der medizinisch fachlichen Expertise auch ethische Handlungsempfehlungen mit einbeziehen $z u$ können. Ziel dieses Kapitels soll es daher sein, den Fokus auf diesen wichtigen Bereich der Notfallmedizin zu richten. 


\begin{abstract}
bekleidet im Bett und ruft um Hilfe. Sie versucht aus dem Bett zu steigen, wird daran durch die Besatzung des RTW, die einige Minuten zuvor eingetroffen war, wiederholt gehindert. Die Rettungsassistentin des kurz vor dem NEF eingetroffenen RTW berichtet, dass die Patientin offenbar verwirrt sei, der Blutdruck betrage $210 \mathrm{zu} 100 \mathrm{mmHg}$, das Anlegen eines EKG sei am Widerstand der Patientin gescheitert. Außerdem bittet sie den Notarzt, dafür zu sorgen, dass alle nicht an der medizinischen Versorgung beteiligten Einsatzkräfte das Schlafzimmer verlassen. In der nicht sehr großen Wohnung der Patientin, die sie seit dem Tode ihres Mannes vor einem Jahr sonst allein bewohnt, befinden sich zu diesem Zeitpunkt folgende Personen: zwei Polizisten, drei Feuerwehrleute, ein Nachbar, drei Rettungsassistenten und ein Notarzt. Polizei und Feuerwehr waren hinzugezogen worden, um die Wohnung zu öffnen. Es wurde vermutet, der Patientin sei etwas zugestoßen, da sie sich entgegen ihrer festen Gewohnheit nicht bei der in einer anderen Stadt lebenden Tochter telefonisch gemeldet hatte und auch selbst nicht am Telefon zu erreichen gewesen war.

Es bedarf mehrerer Aufrufe, bis im Schlafzimmer bei der Patientin nur noch die Rettungsassistentin und der Notarzt verbleiben. Zwischenzeitlich trifft die Tochter ein und fragt barsch, weshalb die Patientin noch nicht in die Klinik transportiert worden sei. In diesem Moment gelingt es der Patientin, die Schublade ihres Nachttisches zu öffnen und das darin befindliche Hörgerät in Betrieb zu nehmen. Die Tochter bestätigt, dass ihre Mutter altersschwerhörig sei. Das Hörgerät erlaubt nun, mit der Patientin in Kontakt zu treten. Sie berichtet, dass sie plötzlich aus dem Schlaf gerissen wurde und ihre Wohnung voller fremder Leute fand. Sie fürchtete, in ihrer Wohnung sei eingebrochen worden. Nach kurzem Gespräch und Klärung der Situation geht es der Patientin deutlich besser, Blutdruck und Puls normalisieren sich und sie lehnt einen Transport in die Klinik vehement ab. Die Tochter widerspricht der Mutter. Sie ist der Auffassung, nur in der Klinik könne die Mutter sicher versorgt werden. Es bedarf eines ausführlichen Gesprächs, bis auch die Tochter die Entscheidung der Mutter akzeptiert.
\end{abstract}

Dieses Fallbeispiel zeigt, wie schnelles und von hoher Motivation getragenes Handeln dazu führen kann, die Würde und den Willen eines Menschen zu missachten. Veranlasst durch die verständliche Sorge der Tochter wird eine Handlungskette angestoßen, an deren Ende zehn Personen in die Wohnung einer zurückgezogen lebenden Witwe eindringen und diese aus dem Schlaf reißen. Wie konnte es dazu kommen?

Seit einigen Jahren versucht man in der Medizin, durch den Einsatz von Algorithmen Entscheidungs- und Handlungsabläufe zu standardisieren. Dies geschieht unter der Vorstellung, dass Algorithmen in kritischen, von hohem Zeitdruck gekennzeichneten Situationen mehr Sicherheit und Effizienz bieten können. Als Vorbild dienen dabei Algorithmen und Entscheidungsbäume, wie sie bei der Programmierung von Computern verwendet werden. Im Gegensatz zum Computer, dessen MultitaskingFähigkeiten noch sehr beschränkt sind, vermag das menschliche Gehirn jedoch sehr gut, parallele Handlungsebenen abzuarbeiten. Ohne Zweifel helfen Algorithmen, bei komplexeren Handlungsabfolgen einen sicheren und vollständigen Ablauf zu gewährleisten. So werden solche Algorithmen in der Notfallmedizin beim Herz-Kreislauf-Stillstand, der Versorgung von Schwerverletzten sowie im Rahmen der erweiterten Kompetenzen von Rettungsassistenten und Notfallsanitätern sinnvoll eingesetzt. Ergänzend dazu wäre es folgerichtig, auch einen „ethischen Algorithmus“ zu entwickeln. Salomon stellte 2009 ein Schema vor, das die wesentlichen Fragen zu ethischen Entscheidungen zusammenfasst. Basierend darauf soll im Folgenden ver- 


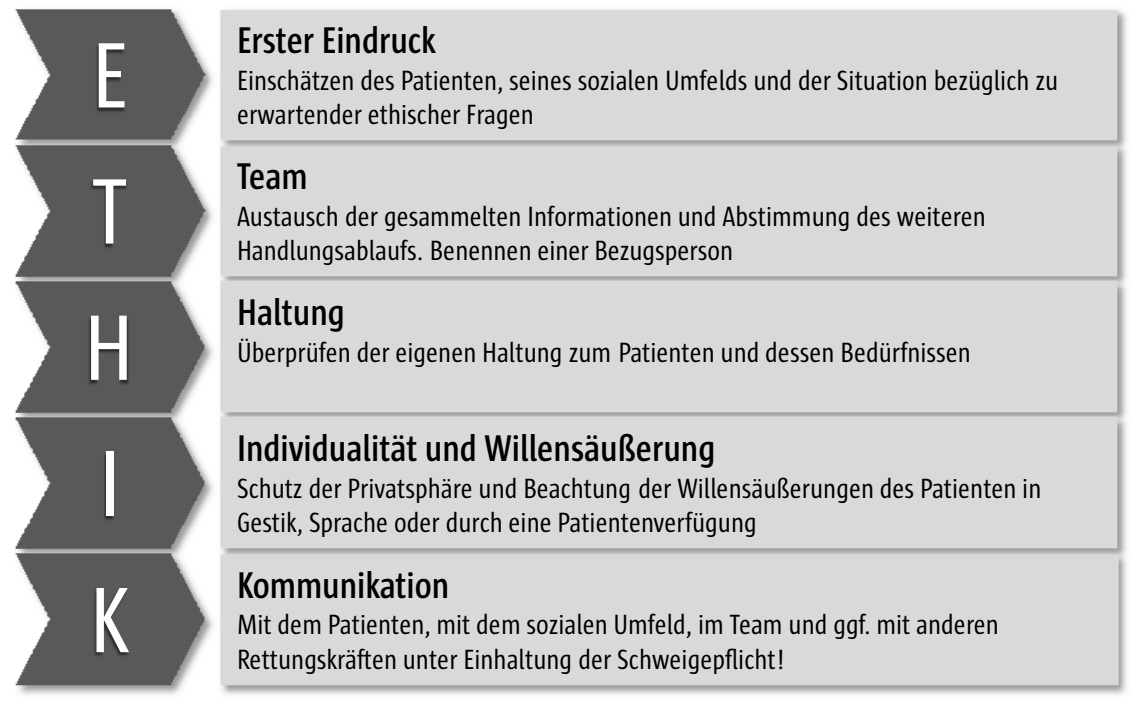

Abb. 2 Algorithmus Ethik in der Notfallmedizin. Dieser Algorithmus soll parallel zu medizinisch notwendigen Maßnahmen ablaufen und stellt sicher, dass alle wesentlichen Eckpunkte, die zur Würde des Patienten beitragen können, beachtet werden.

sucht werden, die Bausteine eines solchen Algorithmus vorzustellen und diese sodann in einen mnemotechnischen Bezug zu setzen, der auch im Notfall anwendbar wäre (s. Abb. 2).

\subsection{Erster Eindruck}

Wenn man die Erfahrungen zahlreicher Notfalleinsätze reflektiert, stellt man häufig fest, dass bereits ab der Einsatzmeldung ein Prozess beginnt, sich ein Bild von der zu erwartenden Lage zu machen. Am Einsatzort angekommen entsteht daraus dann ein Erster Eindruck, der hilfreich sein kann, die richtigen Prioritäten zu setzen. Im Fallbeispiel war die Räumung der Wohnung ein solcher Aspekt. Diese Maßnahme war medizinisch wie ethisch begründet, da zum einen anders keine Untersuchung der Patientin sinnvoll möglich, vor allem aber die Intimsphäre der Patientin zu schützen war. Bei der Behandlung von Notfallpatienten spielt der Faktor Zeit eine große Rolle. Durch qualifiziertes Personal und die medikamentös wie technisch hochwertige Ausstattung moderner Rettungsmittel können zahlreiche diagnostische und therapeutische Maßnahmen noch am Einsatzort durchgeführt werden, die sonst der Klinik vorbehalten waren. Dennoch gilt nach wie vor das Prinzip der sogenannten Golden Hour, nach der ein Patient das Krankenhaus erreicht haben sollte. Von daher steht die Priorisierung der notwendigen Maßnahmen im Interesse des Patienten an erster Stelle. Andererseits besteht das Rettungsteam aus mehreren Personen, sodass ethisch begründete Maßnahmen auch begleitend durchführbar sind. Selbstverständlich dürfen die medizinisch unverzüglich erforderlichen Maßnahmen bei vitaler Bedrohung dadurch nicht verzögert werden. 


\subsection{Team}

Mit dem Rettungsteam sollten gemeinsam Informationen gesammelt und der Erste Eindruck sowie die daraus abgeleitete Priorisierung der weiteren Maßnahmen so früh wie möglich besprochen werden. Diese Abstimmung ist unerlässlich, um einen koordinierten Handlungsablauf zu gewährleisten und die Aufgaben sinnvoll zu verteilen. Neben den „technischen“ Dingen (Diagnostik, Vorbereiten von Medikamenten) sollte eine Person den Kontakt zum Patienten halten. Der Patient hat dadurch einen festen Ansprechpartner und kann Auskunft geben, aber auch eigene Fragen stellen. Die Erfahrung zeigt, dass dieses Vorgehen Zeit spart, wichtige Informationen sammelt und dem Patient das Gefühl vermittelt, nicht „versorgt“, sondern vor allem umsorgt zu sein.

\subsection{Haltung}

Ethisches Handeln erfordert eine besondere Haltung, um ethische Prinzipien zu wahren. Gerade Notfalleinsätze entwickeln mitunter eine Eigendynamik, in der zwar die beste medizinische Behandlung als Ziel erreicht wird, Autonomie und Würde des Patienten jedoch auf der Strecke bleiben. Um das zu verhindern, bedarf es einer Haltung, die ethische wie medizinische Aspekte erkennt und auf die individuellen Bedürfnisse des jeweiligen Patienten abstimmt. Diese Selbstbestimmung leitet sich aus der durch das Grundgesetz geschützten Würde und Freiheit des Menschen ab.

Jeder medizinisch indizierte Eingriff bedarf daher zwingend der Einwilligung des Patienten. Der Patient kann jederzeit seine Einwilligung widerrufen oder die Fortsetzung der Behandlung ablehnen. Ist der Patient nicht zu einer klaren Willensbekundung in der Lage, ist nach dessen mutmaßlichem Willen zu handeln (vgl. Kap. 8 „Wahrung der Patienten-Selbstbestimmung in der Notfallmedizin durch vorausschauende Behandlungsplanung [Advance Care Planning]“). Im geschilderten Fallbeispiel bedarf die Rolle der Tochter der Patientin einer genaueren Betrachtung. Sie hatte ihre Mutter telefonisch nicht erreichen können und war daher in großer Sorge über deren Zustand. Der von ihr kontaktierte Nachbar erhielt auf Klingeln und Klopfen keine Reaktion der Patientin. Somit ist die Wohnungsöffnung durch die Feuerwehr eine sinnvolle Maßnahme für den vermuteten Notfall einer hilflos in der Wohnung liegenden alleinstehenden Dame. Die später am Notfallort eintreffende Tochter drängte sehr auf eine Klinikeinweisung ihrer Mutter. So ergibt sich einmal mehr eine potenzielle Konfliktsituation zwischen Patientin und deren Angehörigen. Aufgabe des Rettungsteams ist in diesen Fällen vor allem, eine möglichst einvernehmliche, aber dem Patientenwillen entsprechende Lösung herbeizuführen. Im konkreten Fall konnte die Tochter überzeugt werden, dass der Wunsch der Mutter, zuhause zu bleiben, nicht nur verständlich, sondern auch aus medizinischer Sicht vertretbar sei. Selbst wenn die medizinische Situation der Patientin nicht völlig stabil gewesen wäre, hätte der Wille der Patientin, zuhause zu bleiben, berücksichtigt werden müssen. Ein Abweichen vom ausdrücklichen Wunsch der Patienten ist nur dann in Erwägung zu ziehen, wenn Zweifel an deren Urteils- und Willensbildungsfähigkeit gegeben sind oder wenn sich konkrete Anhaltspunkte dafür ergeben haben, dass möglicherweise sogar eine Eigen- oder Fremdgefährdung vorliegt. Gerade bezüglich der Eigengefährdung ist eine sorgfältige Prüfung der Urteilsfähigkeit eines Patienten unerläss- 
lich, um sicherzugehen, dass es sich tatsächlich um eine freie und fundierte Willensbildung und nicht um eine Kurzschlussreaktion handelt. Diese Differenzierung ist auch für den erfahrenen Notarzt, der den Patienten in der Regel erst seit wenigen Minuten kennt, oft extrem schwierig. Hier kann es erforderlich sein, eine fachliche Begutachtung durch einen Psychiater herbeizuführen. Dies wird in aller Regel nur durch den Transport in eine Klinik mit entsprechendem Konsildienst möglich sein.

\subsection{Individualität und Willensäußerung}

Notfallsituationen finden nicht selten im öffentlichen Raum statt und ziehen damit das Interesse vieler Passanten auf sich. Dennoch gilt es auch hier, die Privatsphäre eines Patienten so weit wie irgend möglich zu schützen. Bereits in dem geschilderten Fallbeispiel in der Wohnung einer Patientin wurde wiederholt ohne triftigen Grund die Privatsphäre durchbrochen. Man möge sich einmal vor Augen führen, wie die Situation aus Sicht der Patientin empfunden wurde. Sie wird in ihrem Bett spät abends durch Schütteln an der Schulter von einer ihr unbekannten Person geweckt. In ihrem Schlafzimmer sieht sie fünf weitere ihr völlig unbekannte Personen. Sie hört die ihr gestellten Fragen nicht. Ihr Versuch, das im Nachttisch befindliche Hörgerät einzusetzen, wird vermutlich aus Angst, dass sie aus dem Bett fallen könne, verhindert. Dass sie in einer solchen Situation den Versuch der Rettungsassistentin, ein EKG anzulegen, unterbindet, ist angesichts der Anwesenheit ihr fremder Personen durchaus verständlich. Möglicherweise wäre die Situation für die Patientin und das Rettungsteam frühzeitig entspannt worden, wenn alle nicht zur medizinischen Versorgung notwendigen Personen früher aus dem Raum geschickt worden wären. Des Weiteren ist es oft hilfreich, wenn eine Person gleichen Geschlechts (in diesem Fall die Rettungsassistentin) die Maßnahmen durchführt, die das Schamgefühl der Patientin berühren. Schließlich wäre es möglich gewesen, den Grund zu erfragen, warum sich die Patientin aus dem Bett erheben wollte.

In einem Gebäude lässt sich ein Notfallpatient meistens gut vor der Öffentlichkeit abschirmen. Anders ist die Lage bei einem Notfallort im Freien, beispielsweise bei einem Verkehrsunfall oder bei einem Notfall im Rahmen einer Großveranstaltung, wo zunächst keinerlei bauliche Barrieren den Blick auf den Patienten verstellen. Ein zunehmendes Problem stellt darüber hinaus der sogenannte Notfalltourismus Schaulustiger dar. Informiert durch Internet oder Verkehrsfunk suchen diese Menschen gezielt Notfallorte auf, um „live“ dabei zu sein. Das rücksichtslose Verhalten dieser Gaffer geht so weit, dass sie den Rettungseinsatz massiv behindern, beispielsweise indem sie so nahe an die Einsatzkräfte rücken, dass kaum noch Raum für die Notfallmaßnahmen bleibt.

Ein wirksames Mittel, um hier Entlastung zu erlangen, ist die gezielte Ansprache einzelner Personen und deren Einbindung in die Rettungsmaßnahmen: „Würden Sie bitte diese Infusion hochhalten!!“. Nimmt der Angesprochene den Auftrag an, dann weichen die Übrigen ein paar Schritte zurück, um nicht als nächste involviert zu werden. Lautstarke Belehrungen schlagen meist fehl und finden nicht selten Veröffentlichung im Internet, da gerade in solchen Einsatzsituationen zahlreiche Videoclips mit den omnipräsenten Smartphones aufgezeichnet werden. In Abänderung des Kant'schen kategorischen Imperativs könnte man heute formulieren: Handle stets so, dass dein Tun als Video aufgezeichnet und im Internet veröffentlicht werden kann. 
Jeder invasive medizinische Eingriff setzt die wirksame Aufklärung sowie das Einverständnis des Patienten voraus. Diese Regel gilt auch bei Notfallpatienten. Ist der Patient nicht einwilligungsfähig, beispielsweise weil er die Tragweite seiner Entscheidung nicht absehen kann oder seine Urteilsfähigkeit infrage steht, ist der mutmaßliche Wille eines urteilsfähigen und die Tragweite der Entscheidung erfassenden Patienten zu unterstellen. Hat der Patient eine Patientenverfügung erstellt, in der für den konkreten Fall einschlägige Anweisungen enthalten sind, ist diese zu berücksichtigen. Liegt ein vital bedrohlicher Notfall vor, der unmittelbares Handeln erfordert, ist es meist nicht vertretbar, wertvolle Zeit mit der Bewertung der vorgelegten Patientenverfügung sowie der Sicherung der korrekten Identität des Verfügenden zu vertun. In einem solchen Fall sind Maßnahmen zu ergreifen, die möglicherweise zu einem späteren Zeitpunkt in Kenntnis der detaillierten Vorgaben der Patientenverfügung und Nachversicherung der Identität wieder revidiert werden müssen. Ein solches Handeln ist aus ethischer Sicht insofern vertretbar, als im umgekehrten Fall wertvolle Zeit verschenkt wird, ohne dass im Vorhinein bekannt ist, ob der Patient durch seine Verfügung eben diesen Maßnahmen zustimmt oder diese ablehnt. Mithin würde die mit der Bewertung der Patientenverfügung verbundene Verzögerung immer dann dem Willen des Patienten widersprechen, wenn sich in der Patientenverfügung kein Hinweis darauf findet, dass die konkreten Maßnahmen vom Patienten nicht gewünscht werden. Aus ethischer und juristischer Sicht ist es gerechtfertigt, auf die in der Notfallsituation nach Standards oder Algorithmen eingeleiteten Maßnahmen später wieder zu verzichten, wenn der Informationsstand eine angemessenere Beurteilung zulässt. Dies entspricht der in der Medizinethik heute vorherrschenden und juristisch unterstützten Auffassung, dass nicht der Abbruch einer Maßnahme begründet werden, sondern die Indikation für ihre Durchführung gegeben sein muss. Besteht keine Indikation (mehr) oder mangelt es an einer wirksamen Einwilligung durch den Patienten, ist die Weiterführung nicht mehr gerechtfertigt (vgl. Positionspapier der DIVI zur Therapiebegrenzung und Therapiezieländerung [Janssens et al. 2012]).

Immer mehr Menschen lehnen unter bestimmten Voraussetzungen die Einleitung von Wiederbelebungsmaßnahmen ab. Dies kann durch eine entsprechende Passage in der Patientenverfügung oder auch durch ein kurzes Dokument geschehen, das der Patient stets bei sich trägt. Mitunter enthält auch die Akte in stationären Pflegeeinrichtungen einen sogenannten DNR „Do-not-resuscitate“- oder, zutreffender, DNAR „Do-not-attempt-resuscitation“-Hinweis. Auf jeden Fall muss sorgfältig auf die Übereinstimmung der Identität geachtet werden. Im Zweifel ist unverzüglich mit Reanimationsmaßnahmen zu beginnen.

\subsection{Kommunikation}

Die schwerhörige Patientin des Fallbeispiels zeigt, welchen Einfluss Kommunikationsprobleme auf die Interaktion zwischen Patient und Einsatzkräften hatten. Dabei dient die Kommunikation nicht allein dem Austausch von Fakten (Sachebene), vielmehr hat die Kommunikation mit Patienten auch therapeutische Aspekte (Angst und Stress lindern durch Information, Trost, Mitgefühl). Störungen der Kommunikation haben vielfältige Ursachen: Hörvermögen, Sprechstörung, fehlendes Gebiss, Einschränkung der zerebralen Leistungsfähigkeit durch somatische und/oder psychische Erkrankung. 
Es sollte auf jeden Fall versucht werden, die bestehende Störung zu erkennen und zu kompensieren. So ist beispielsweise eine Kommunikation mit einem an Amyotropher Lateralsklerose erkrankten Patienten, der nahezu vollständig gelähmt ist, noch über Augenzwinkern oder andere eindeutige motorische Reaktionen möglich, sofern die Fragen so gestellt werden, dass sie mit ja oder nein zu beantworten sind. Gerade in kritischen Situationen, die für den Patienten auch eine hohe emotionale Beteiligung bewirken, hat die nonverbale Kommunikation besonderes Gewicht. Allein die Haltung, die Gestik und Mimik, mit der man einem Patienten gegenübertritt, können Vertrauen wecken und beruhigen oder aber Unsicherheit und Angst steigern und sogar Aggressivität auslösen. Es erfordert Erfahrung und Einfühlungsvermögen, um in der jeweiligen Situation angemessen reagieren zu können. Dazu zählt im Einzelfall auch das Halten der Hand oder ein In-den-Arm-Nehmen des Patienten.

Innerhalb des Teams ist eine klare Kommunikation von zentraler Bedeutung, um Missverständnisse $\mathrm{zu}$ vermeiden und vollständige Informationen zu sammeln. Mutmaßungen oder nicht weitergegebene Informationen können zu falschen Beurteilungen und damit zu Fehlentscheidungen führen.

Die Abbildung des dargestellten Ethik-Algorithmus (s. Abb. 2) soll helfen, in einer Notfallsituation die wesentlichen Elemente ethischen Handelns nicht zu übersehen. Er ist bewusst nicht in die Abfolge medizinischer Algorithmen eingefügt, da er wie eingangs beschrieben parallel zu den übrigen Maßnahmen gesehen werden muss. Der erste Schritt dieses Algorithmus ist folgerichtig die Priorisierung der Maßnahmen. Damit soll vermieden werden, dass dringende medizinische Maßnahmen verzögert werden, andererseits ist bei jedem Schritt zu prüfen, inwieweit die Maßnahme ethisch vertretbar ist. Dabei spielen die Autonomie und der möglicherweise auch schriftlich festgelegte Wille des Patienten eine entscheidende Rolle. Die Beachtung der im Algorithmus zusammengefassten Elemente stellt auch beim Notfallpatienten sicher, dass dessen Würde respektiert wird.

\section{Literatur}

Janssens U, Burchardi H, Duttge G, Erchinger R, Gretenkort P, Mohr M, Nauck F, Rothärmel S, Salomon F, Schmucker P, Simon A, Stopfkuchen H, Valentin A, Weiler N, Neitzke G (2012) Therapiezieländerung und Therapiebegrenzung in der Intensivmedizin. Positionspapier der Sektion Ethik der DIVI. http://www.divi.de/images/ Dokumente/Empfehlungen/Therapiezielaenderung/Positionspapier_Ethik_2012.pdf (Zugriff am 20.08.2015)

Salomon F (2009) Ethische Aspekte der Entscheidungsfindung. In: Madler C, Jauch K-W, Werdan K, Siegrist I, Pajonk F-G (Hrsg.) Akutmedizin - Die ersten 24 Stunden. Das NAW Buch. Elsevier, S. 161-167

Srivastava R (2013) Speaking Up - When Doctors Navigate Medical Hierarchy. New England Journal of Medicine 368(4): 302-305

Stern R, Arntz H-R (2012) Ethik und Notfallmedizin. Notfall + Rettungsmedizin 15(8): 657-674 


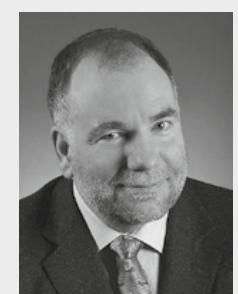

\section{PD Dr. med. Heiner Krieter, DEAA}

1980-1982 Studium der Physik in Münster. Studium der Humanmedizin 19811987 an der FU Berlin und der Medizinischen Universität zu Lübeck. 1987-1989 Studium Medizintechnik/Physikalische Technik an der FH Lübeck. 1988-1990 Wissenschaftlicher Angestellter des Instituts für Anästhesiologie der Medizinischen Universität zu Lübeck (Prof. Dr. van Ackern). Forschung an der Abteilung für Experimentelle Chirurgie der Universität Heidelberg (Prof. Dr. Meßmer). Promotion 1992. 1991-2005 Arzt an der Klinik für Anästhesiologie und Operative Intensivmedizin der Medizinischen Fakultät Mannheim der Universität Heidelberg (Prof. Dr. van Ackern). 1998 Facharzt für Anästhesiologie. 1999 Diplom der European Academy of Anaesthesiology, DEAA. 2003 Habilitation. 2002-2005 Leitender Oberarzt des Bereichs Notfallmedizin am Universitätsklinikum Mannheim. 2005-2008 Chefarzt der Abteilung für Anästhesiologie, Operative Intensivmedizin, Notfallmedizin und Schmerztherapie einer Klinik im Saarland. Lehrtätigkeit im Fach Anästhesiologie an der Medizinischen Fakultät Mannheim. Regelmäßige Tätigkeit als Flugarzt bei der DRF. Kreisverbandsarzt und Ärztlicher Verantwortlicher Rettungsdienst beim DRK Kreisverband Mannheim. Seit 2008 Gemeinschaftspraxis mit Frau Dr. Michaela Weiß in Mannheim niedergelassen. Schwerpunkte: Anästhesiologie, Schmerztherapie und Palliativmedizin. Gründungsmitglied des Mannheimer Netzwerks „PalMa“ für die spezialisierte ambulante Palliativversorgung (SAPV). Gemeinsam mit Frau Dr. Michaela Weiß Leiter der Palliativmedizinischen Akademie in Mannheim. 